\title{
Increased osteoclastic activity in acute Charcot's osteoarthopathy: the role of receptor activator of nuclear factor-kappaB ligand
}

\author{
G. Mabilleau • N. L. Petrova • M. E. Edmonds • \\ A. Sabokbar
}

Received: 19 December 2007 / Accepted: 22 February 2008 / Published online: 4 April 2008

(C) Springer-Verlag 2008

\begin{abstract}
Aims/hypothesis Our aims were to compare osteoclastic activity between patients with acute Charcot's osteoarthropathy and diabetic and healthy controls, and to determine the effect of the receptor activator of nuclear factor-kappaB ligand (RANKL) and its decoy receptor osteoprotegerin (OPG).

Methods Peripheral blood monocytes isolated from nine diabetic Charcot patients, eight diabetic control and eight healthy control participants were cultured in the presence of macrophage-colony stimulating factor (M-CSF) alone, M-CSF and RANKL, and also M-CSF and RANKL with excess concentrations of OPG. Osteoclast formation was assessed by expression of tartrate-resistant acid phosphatase on glass coverslips and resorption on dentine slices.

Results In cultures with M-CSF, there was a significant increase in osteoclast formation in Charcot patients compared with healthy and diabetic control participants $(p=0.008)$. A significant increase in bone resorption was also seen in the former, compared with healthy and diabetic control participants $(p<0.0001)$. The addition of RANKL to the cultures with M-CSF led to marked increase in osteoclastic resorption in Charcot (from $0.264 \pm 0.06 \%$ to
\end{abstract}

G. Mabilleau $\cdot$ A. Sabokbar

Nuffield Department of Orthopaedic Surgery,

Botnar Research Centre, University of Oxford,

Oxford, UK

N. L. Petrova $\cdot$ M. E. Edmonds $(\square)$

Diabetic Foot Clinic,

King's College Hospital NHS Foundation Trust,

Denmark Hill,

London SE5 9RS, UK

e-mail: Michael.Edmonds@kch.nhs.uk
$41.6 \pm 8.1 \%, p<0.0001)$ and diabetic control $(0.000 \pm 0.00 \%$ to $14.2 \pm 16.5 \%, p<0.0001)$ patients, and also in healthy control participants $(0.004 \pm 0.01 \%$ to $10.5 \pm 1.9 \%, p<0.0001)$. Although the addition of OPG to cultures with M-CSF and RANKL led to a marked reduction of resorption in Charcot patients $(41.6 \pm 8.1 \%$ to $5.9 \pm 2.4 \%, p=0.001)$, this suppression was not as complete as in diabetic control patients $(14.2 \pm 16.5 \%$ to $0.45 \pm 0.31 \%, p=0.001)$ and in healthy control participants (from $10.5 \pm 1.9 \%$ to $0.00 \pm 0.00 \%$, $p<0.0001$ ).

Conclusions/interpretation These results indicate that RANKL-mediated osteoclastic resorption occurs in acute Charcot's osteoarthropathy. However, the incomplete inhibition of RANKL after addition of OPG also suggests the existence of a RANKL-independent pathway.

Keywords Charcot's osteoarthropathy · OPG · Osteoclasts . Osteolysis $\cdot$ RANKL $\cdot$ Resorption
Abbreviations
LIGHT homologous to lymphotoxins exhibiting in- ducible expression and competing with herpes simplex virus glycoprotein D for herpes virus entry mediator (HVEM), a receptor expressed by $\mathrm{T}$ lymphocytes
MEM minimum essential medium
M-CSF macrophage-colony stimulating factor
OPG osteoprotegerin
PBMCs peripheral blood monocytes
RANK receptor activator of nuclear factor-kappaB
RANKL receptor activator of nuclear factor-kappaB ligand
sRANKL soluble receptor activator of nuclear factor- kappaB ligand
TRAcP tartrate-resistant acid phosphatase 


\section{Introduction}

Although Charcot's osteoarthropathy is characterised by increased local bone resorption [1], the exact cellular mechanisms contributing to the pathogenesis of this condition remain unresolved. Osteoclasts have been shown to be the principal cell type responsible for bone resorption [2]. These cells originate from the haemopoietic lineage and are known to undergo various stages of proliferation, fusion and differentiation before they are fully functionally active, mature osteoclasts. Recently, receptor activator of nuclear factor-kappaB (RANK) ligand (RANKL) has been identified as an essential mediator of osteoclast formation and activation [3]. RANKL is expressed on a variety of cell types such as bone forming osteoblasts, T lymphocytes, dendritic cells, endothelial cells and fibroblasts. RANKL mediates the process of osteoclastogenesis by binding to its RANK, which is expressed on mononuclear osteoclast precursors. The effects of RANKL-RANK interaction are physiologically counterbalanced by osteoprotegerin (OPG), which acts as a soluble receptor decoy for RANKL and blocks the interaction of RANKL with RANK. The ratio of RANKL to OPG has been suggested to regulate the extent of osteoclast formation and resorption. Therefore, any alteration in the RANKL/OPG ratio could be critical in the pathogenesis of osteolytic bone disorders [4].

Recently, Jeffcoate hypothesised that the RANK/RANKL/ OPG pathway may play an important role in the osteolysis seen in acute Charcot's osteoarthropathy [5]. Using an in vitro technique to generate functional human osteoclasts from peripheral blood monocytes (PBMCs) [6] in the presence of macrophage-colony stimulating factor (M-CSF) [7] and soluble RANKL, it is possible to determine the cellular mechanisms involved in the process of osteoclast formation and resorption in physiological and pathological conditions. To our knowledge, this technique has not yet been studied in patients with Charcot's osteoarthropathy.

The aims of this study were: (1) to generate functional human osteoclasts in vitro from diabetic patients with acute Charcot's osteoarthropathy and from healthy and diabetic control participants; (2) to compare the extent of osteoclast formation and resorption; and (3) to determine the role of the RANK/RANKL/OPG pathway in osteoclastic activity in Charcot's osteoarthropathy.

\section{Methods}

\section{Patients}

We studied nine consecutive diabetic patients with recent onset of acute Charcot's osteoarthropathy (five men, four women; five type 1 , four type 2 diabetes), eight diabetic patients with no previous history of Charcot's osteoarthropathy (five men, three women; four type 1, four type 2) and eight healthy control participants (five men, three women). Patients with acute Charcot's osteoarthropathy were matched for age and duration of diabetes with the diabetic control patients and for age with the healthy control participants. The mean age was similar between patients with Charcot's osteoarthropathy and diabetic control patients $(53 \pm 2.8$ versus $59 \pm 2.9$ years $[$ mean $\pm \mathrm{SEM}], p=0.167)$ as was the mean age between the former and healthy control participants $(53 \pm 2.8$ versus $47 \pm 2.7$ years, $p=0.114$ ). The mean duration of diabetes was similar in both groups with diabetes $(31 \pm 5.1$ [Charcot patients] versus $27 \pm 4.6$ years, $p=0.606)$. Diabetes control as indicated by glycated $\mathrm{Hb}$ was also similar in the two diabetes groups (7.7 \pm 0.6 [Charcot's] versus $7.8 \pm 0.4 \%, p=0.743)$.

Diagnosis of Charcot's osteoarthropathy was made on the presentation of a hot swollen foot, with skin foot temperature $2^{\circ} \mathrm{C}$ greater than the corresponding site on the contralateral foot and with typical radiological changes of subluxation, dislocation or fragmentation of bone on standard foot radiographs [8]. All patients had intact feet and no evidence of foot infection or ulceration.

Ethical permission for this study was obtained from the King's College Hospital Research Ethics Committee and all participants gave written informed consent.

\section{Isolation and culture of monocytes}

Peripheral blood mononuclear cells were isolated as previously described [6]. Briefly, blood was diluted $1: 1$ in $\alpha$-minimum essential medium (MEM; Invitrogen, Paisley, $\mathrm{UK}$ ), layered over Histopaque and centrifuged (693 $g$ ) for $20 \mathrm{~min}$. The interface layer was resuspended in MEM, then centrifuged $(600 \mathrm{~g})$ for a further $10 \mathrm{~min}$. The resultant cells were resuspended in MEM with 10\% heat-inactivated FCS and counted in a haemocytometer following lysis of erythrocytes by a 5\% (vol./vol.) acetic acid solution.

To assess the extent of osteoclast formation and resorption, PBMCs were cultured on glass coverslips and dentine slices. Initially, $5 \times 10^{5}$ PBMCs were added to 6-mm diameter glass coverslips and 4-mm diameter dentine slices in MEM containing $100 \mathrm{UI} / \mathrm{ml}$ penicillin, $100 \mu \mathrm{g} / \mathrm{ml}$ streptomycin and 10\% FCS (Gibco, Paisley, UK). After $2 \mathrm{~h}$ incubation, coverslips and dentine slices were vigorously rinsed in medium to remove non-adherent cells. The cultures were maintained in MEM/FCS under three different culture conditions: (1) human M-CSF (R\&D Systems Europe, Abingdon, UK) alone at $25 \mathrm{ng} / \mathrm{ml}$; (2) M-CSF plus $100 \mathrm{ng} / \mathrm{ml}$ human soluble RANKL (sRANKL; Peprotech, London, UK) (a concentration known to facilitate differentiation of osteoclast precursors to active bone-resorbing osteoclasts in vitro); and (3) M-CSF plus sRANKL plus $250 \mathrm{ng} / \mathrm{ml}$ human OPG (R\&D Systems Europe). 
Coverslips and dentine slices were cultured at $37^{\circ} \mathrm{C}$ in $5 \% \mathrm{CO}_{2}$ for 14 and 21 days respectively.

\section{Osteoclast formation}

After 14 days, the coverslips were examined histochemically for the expression of tartrate-resistant acid phosphatase (TRAcP), an osteoclast marker. Coverslips with newly formed osteoclasts were collected and rinsed in PBS buffer, fixed with formalin (10\% [vol./vol.] in PBS buffer) for $10 \mathrm{~min}$ and rinsed in distilled water. TRAcP was histochemically revealed by a simultaneous coupling reaction using Naphtol AS-BI-phosphate as substrate and Fast violet $\mathrm{B}$ as the diazonium salt. The coverslips were incubated for $90 \mathrm{~min}$ at $37^{\circ} \mathrm{C}$ in a dark room, rinsed three times in distilled water and the residual activity was inhibited by $4 \% \mathrm{NaF}$ (wt/wt) for $30 \mathrm{~min}$. Coverslips were then rinsed in distilled water, counterstained with DAPI for $20 \mathrm{~min}$ and allowed to dry before mounting, using an aqueous medium. TRAcP-positive cells with more than three nuclei were identified as osteoclasts. The number of newly generated osteoclasts was assessed using a light microscope examination.

\section{Osteoclast resorption}

After 21 days, the dentine slices were removed from the culture wells, placed in $\mathrm{NH}_{4} \mathrm{OH}(1 \mathrm{~mol} / \mathrm{l})$ for $30 \mathrm{~min}$ and sonicated for $5 \mathrm{~min}$ to remove any adherent cells. They were then rinsed in distilled water and stained with $0.5 \%$ (vol./vol.) toluidine blue prior to examination by light microscopy. The surface of each dentine slice was examined for evidence of lacunar resorption and the extent of eroded surface on each dentine slice was determined using image analysis and expressed as the percentage of surface area resorbed.

\section{Statistical analyses}

Data were expressed as a mean \pm SEM. Initially the difference within the three study groups (Charcot patients, healthy and diabetic controls) was assessed with the non-parametric Kruskall-Wallis test. Then the differences between Charcot and diabetic patients, and Charcot patients and healthy controls were assessed by the non-parametric Mann-Whitney $U$ test. In each patient group, the differences between the various culture conditions were also assessed using the Mann-Whitney $U$ test. Differences were considered significant at $p<0.05$.

\section{Results}

Osteoclast cultures in the presence of M-CSF

Osteoclast formation The mean number of newly formed TRAcP-positive multinucleated osteoclasts in the presence of M-CSF alone was significantly greater in the patients with acute Charcot's osteoarthropathy $(48.6 \pm 18.2)$ than in diabetic $(6.8 \pm 2.7)$ and healthy control participants $(5.0 \pm 0.7)$ $(p=0.008)$. The number of TRAcP-positive multinucleated osteoclasts formed in acute Charcot's osteoarthropathy was 7.2 and 9.7 times greater than those formed in diabetic ( $p=0.010)$ and healthy control groups $(p=0.003)$, respectively.

Osteoclast resorption The newly formed osteoclasts exhibited increased functional activity as demonstrated by the extent of resorption on dentine slices, with percentage area resorption significantly elevated in the patients with acute Charcot's osteoarthropathy $(0.264 \pm 0.06 \%)$ compared with diabetic $(0.000 \pm 0.00 \%)$ and healthy control groups $(0.004 \pm 0.01) \quad(p<0.0001)$. The percentage of resorption was significantly greater in the Charcot patients than in the diabetic $(p=0.001)$ and healthy control groups $(p=0.001)$.

Osteoclast cultures in the presence of M-CSF and sRANKL

Osteoclast formation The addition of sRANKL led to an increase in the number of TRAcP-positive multinucleated osteoclasts in all three groups of patients. The mean number of these osteoclasts in patients with acute Charcot's osteoarthropathy was $96.0 \pm 21.6$, which was significantly greater than that in the diabetic $(56.5 \pm 11.5)$ and healthy (29.0 \pm 5.1$)$ control groups ( $p=0.010$; Fig. 1a,c,e). The number of TRAcP-positive multinucleated osteoclasts in the patients with acute Charcot's osteoarthropathy was 1.7 times higher than in diabetic control patients, but this finding did not reach significance $(p=0.105)$. However, the number of these osteoclasts in the acute Charcot group was 3.3 times (and significantly) higher than in the healthy control group $(p=0.005)$. When the number of cells in the cultures with M-CSF alone was compared with that after the addition of sRANKL, there was a significant increase in the diabetic control patients (from $6.8 \pm 2.7$ to $56.5 \pm 11.5$, $p=0.003$ ) and in the healthy participants (from $5.0 \pm 0.7$ to $29.0 \pm 5.1, p=0.002$ ), while the increase in the number of TRAcP-positive multinucleated osteoclasts in the acute Charcot group failed to reach significance (increase from $48.6 \pm 18.2$ to $96.0 \pm 21.6, p=0.059$; Fig. 2 a).

Osteoclast resorption The percentage area resorption on dentine slices with M-CSF and sRANKL was significantly increased in the acute Charcot group $(41.6 \pm 8.1 \%)$ compared with that in the diabetic $(14.2 \pm 16.5 \%)$ and healthy control groups $(10.5 \pm 1.9 \% ; p=0.005)$. Resorption in the Charcot patients was 2.9 times higher than in diabetic control patients $(p=0.008)$ and four times higher than in healthy participants $(p=0.005$; Fig. $1 \mathrm{~b}, \mathrm{~d}, \mathrm{f})$. The addition of sRANKL to the cultures with M-CSF led to the 


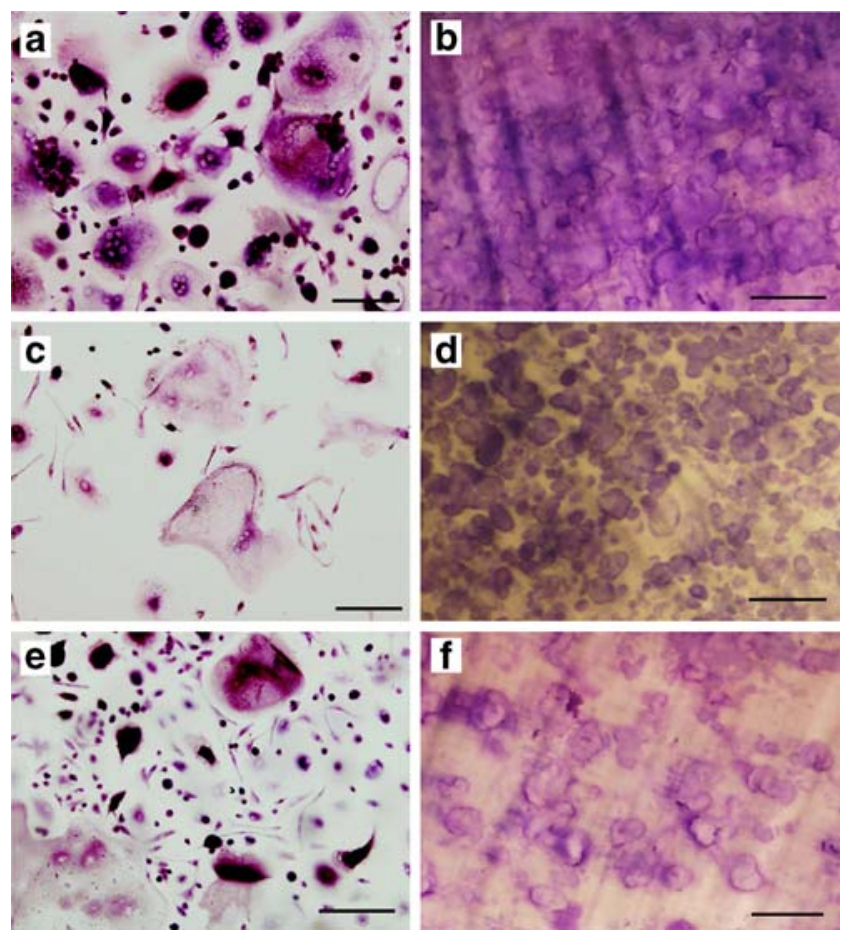

Fig. 1 Multinucleated TRAcP-positive cells were formed on glass coverslips (a, c, e) capable of lacunar resorption (b, d, f) after 14 and 21 days incubation, respectively, in the presence of $25 \mathrm{ng} / \mathrm{ml}$ human M-CSF and $100 \mathrm{ng} / \mathrm{ml}$ sRANKL. Newly formed osteoclasts were numerous and highly active in Charcot's patients $(\mathbf{a}, \mathbf{b})$ compared with diabetic (c, d) and healthy control (e, f) participants. Scale bars, $10 \mu \mathrm{m}$

following rises in the percentage area resorption when compared with M-CSF alone: Charcot's $0.264 \pm 0.06 \%$ to $41.6 \pm 8.1 \%, p<0.0001$; diabetic control $0.000 \pm 0.00 \%$ to $14.2 \pm 16.5 \%, p<0.0001$; healthy control $0.004 \pm 0.01 \%$ to $10.5 \pm 1.9 \%, p<0.0001$ (Fig. 2b).

Osteoclast cultures in the presence of M-CSF, sRANKL and excess concentrations of OPG

Osteoclast formation The addition of excess concentrations of OPG led to a reduction in the number of TRAcP-positive multinucleated osteoclasts in the cultures with M-CSF, sRANKL and OPG in all the three groups of patients. However, after the addition of OPG, the number of TRAcPpositive multinucleated osteoclasts was still significantly increased in the Charcot group (54.4 \pm 17.6$)$, as compared with diabetic $(8.8 \pm 5.3)$ and healthy control participants $(4.4 \pm 1.2 ; p=0.003)$. In the cultures with M-CSF, sRANKL and OPG, the number of TRAcP-positive multinucleated osteoclasts was greater in the Charcot patients than in the diabetic $(p=0.005)$ and healthy control groups $(p=0.001)$.

When OPG was added to the cultures with M-CSF and sRANKL, the reduction in the number of TRAcP-positive

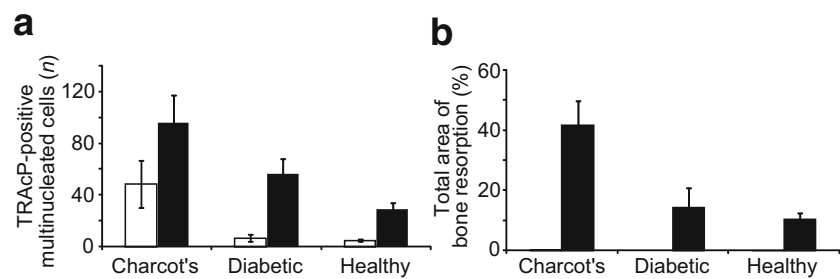

Fig. 2 a Quantitative comparison between the number ( $n$ ) of TRAcPpositive cells formed in cultures with M-CSF alone (white bars) or with M-CSF and sRANKL (black bars) in patients with Charcot's osteoarthropathy and diabetic and healthy control participants. b Quantitative comparison between the percentage area resorption in the same cultures and patient groups. Statistical differences between the groups were determined using the Mann-Whitney $U$ test, with significance as follows: a Charcot's $p=0.059$, diabetic control $p=0.003$, healthy control $p=0.002$; b Charcot's $p<0.0001$, diabetic control $p<0.0001$, healthy control $p<0.0001$

cells in Charcot patients was not significant $(96.0 \pm 21.6$ versus $54.4 \pm 17.6, p=0.189)$. OPG on the other hand significantly inhibited the number of TRAcP-positive cells in M-CSF and RANKL-mediated cultures from diabetic (reduced from $56.5 \pm 11.5$ to $8.8 \pm 5.3, p=0.005$ ) and healthy control participants $(29.0 \pm 5.1$ to $4.4 \pm 1.2, p=0.003$; Fig. 3a).

Osteoclast resorption The addition of OPG led to a marked reduction of the percentage area resorption on dentine slices in Charcot patients (from $41.6 \pm 8.1 \%$ to $5.9 \pm 2.4 \%$, $p=0.001)$ and also in diabetic $(14.2 \pm 16.5 \%$ to $0.45 \pm 0.31 \%$, $p=0.001$ ) and healthy control (from $10.5 \pm 1.9 \%$ to $0.00 \pm 0.00 \%, p<0.0001$ ) participants (Fig. 3b).

However, the percentage area resorption on the dentine slices was still greater in the cultures with M-CSF, RANKL and OPG from the patients with acute Charcot's osteoarthropathy $(5.9 \pm 2.4 \%)$ than in those from diabetic $(0.45 \pm$ $0.31 \%)$ and healthy control $(0.00 \pm 0.00 \%)$ participants

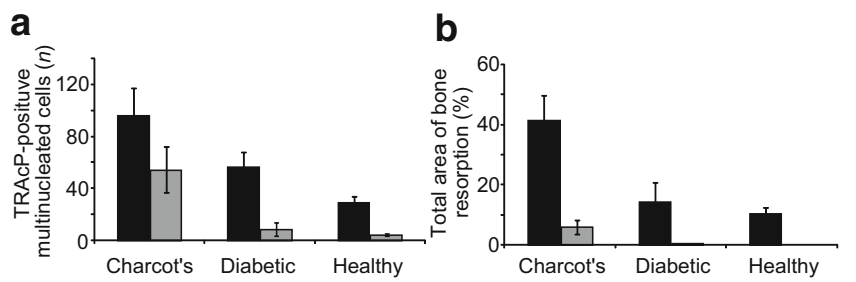

Fig. 3 a Comparison between the number ( $n$ ) of TRAcP-positive cells formed in cultures with M-CSF and sRANKL (black bars) or with M-CSF, sRANKL and excess concentrations of OPG $(250 \mathrm{ng} / \mathrm{ml})$ (grey bars) in patients with Charcot's osteoarthropathy and diabetic and healthy control participants. b Comparison between the percentage area resorption in the same cultures and patient groups. Statistical differences between the groups were determined using the MannWhitney $U$ test, with significance as follows: a Charcot's $p=0.189$, diabetic control $p=0.005$, healthy control $p=0.003$; b Charcot's $p=0.001$, diabetic control $p=0.001$, healthy control $p<0.0001$ 
$(p=0.003)$. Resorption on the dentine slices was greater in the Charcot patients than in diabetic $(p=0.005)$ and healthy control $(p=0.003)$ groups.

\section{Discussion}

This study shows that monocytes from patients with acute Charcot's osteoarthropathy cultured in the presence of M-CSF alone were capable of differentiating into mature osteoclasts that exhibited increased resorption compared with diabetic and healthy control participants. Furthermore, osteoclasts generated after the addition of sRANKL were functionally more aggressive, exhibiting a considerable increase in the extent of resorbing activity in patients with acute Charcot's osteoarthropathy. This resorption was partially blocked by the addition of excess concentrations of OPG, a soluble receptor decoy for RANKL. This suggests that the increased osteoclastic activity in patients with acute Charcot's osteoarthropathy is mediated through both a RANKL-dependent and a RANKL-independent pathway.

Cultures from the patients with Charcot's osteoarthropathy showed increased osteoclast formation and resorption when cultured with M-CSF alone. Although M-CSF is an essential factor for proliferation, differentiation and survival of the monocyte-macrophage lineage $[9,10]$, it is not an osteoclastogenic factor and it is unusual to detect osteoclast formation and resorption in cultures with M-CSF, as was seen in the diabetic and healthy controls. This observation suggests that in acute Charcot's osteoarthropathy there may be increased levels of other circulating pro-inflammatory factors such as TNF- $\alpha$ [11, 12], IL-6 [13], IL-8 [14] and LIGHT (homologous to lymphotoxins exhibiting inducible expression and competing with herpes simplex virus glycoprotein D for herpes virus entry mediator [HVEM], a receptor expressed by $\mathrm{T}$ lymphocytes) [15], which have been previously shown to stimulate osteoclastogenesis independently of RANK/RANKL mechanisms. The concentrations of these circulating factors in diabetic and healthy control participants may not be sufficient to induce the formation and differentiation of active osteoclasts in the presence of M-CSF alone.

After the addition of sRANKL to M-CSF cultures, the newly formed osteoclasts exhibited markedly increased resorption in the patients with Charcot's osteoarthropathy, although the number of osteoclasts did not significantly increase in these patients compared with cultures with M-CSF alone. These observations may not be unique to Charcot's osteoarthropathy, and indeed similar observations have been reported in other conditions associated with increased bone resorption, such as rheumatoid arthritis where the addition of sRANKL resulted in a significant increase in lacunar resorption, but did not lead to a significant increase in the number of TRAcP-positive cells [16]. Overall, the observed extensive resorption in acute Charcot patients, in the presence of M-CSF and sRANKL, as compared with the diabetic or healthy control groups, may suggest that the osteoclast precursors circulating in acute Charcot patients are in a higher activated state and as such are more primed to becoming osteoclasts (mediated through RANKL) than those in the control groups.

In order to ascertain that RANKL was a major osteoclastic activator in patients with Charcot's osteoarthropathy, excess concentrations of OPG, the soluble receptor decoy to RANKL, were added to the cultures with M-CSF and RANKL. The rationale for this approach was that if osteoclastogenesis is mediated solely through RANK-RANKL interaction, addition of excess concentrations of OPG (as had been previously determined to be sufficient to block osteoclastogenesis through RANKL [15]) would completely abolish the process of osteoclast differentiation and activation. In the current study, although osteoclast formation and resorption in the diabetic and healthy control groups was completely blocked by the addition of OPG, the latter did not achieve total inhibition of osteoclast formation and resorption in patients with acute Charcot's osteoarthropathy. These results suggest that although RANKLdependent pathways do play a significant role in the osteoclastic activity of Charcot's osteoarthropathy, an alternative pathway (other than RANK/RANKL) may also be involved. Osteoclastogenic mediators other than RANKL that have been reported to stimulate osteoclast differentiation independently of the RANKL pathway include TNF- $\alpha$ [11, 12], IL-6 [13], IL-8 [14] and LIGHT [15]. In acute Charcot's osteoarthropathy, it is possible that one or a combination of these factors may have initiated the circulating osteoclast precursors to be in a more 'primed' condition, a situation which as such could explain the observed resorption in Charcot monocyte cultures supplemented with M-CSF alone, without the exogenous addition of any osteoclastogenic mediators.

The osteolysis of Charcot's osteoarthropathy may be explained by our observation that osteoclast precursors from Charcot patients develop into mature osteoclasts that exhibit increased resorptive activity, especially in response to RANKL, unlike the increased resorption in response to bacterial infection, which is not mediated by RANKL [17]. Increased expression of RANKL has been previously demonstrated in pathological osteolysis associated with the development of various bone diseases [18] and a similar mechanism may contribute to osteolysis of Charcot's osteoarthropathy [5]. Furthermore, patients with Charcot's osteoarthropathy have severe neuropathy, which itself can also lead to increased expression of RANKL as a result of the loss of nerve-derived peptides known to antagonise its effect such as calcitonin gene-related peptide [5]. In 
addition to the RANKL-dependent pathway, our results suggest that a RANKL-independent pathway, mediated by pro-inflammatory cytokines, may also be important. Indeed, Charcot's foot is characterised by excessive inflammation and proinflammatory cytokines have been implicated in its pathogenesis [19]. In support of this, a recent immunohistochemical analysis of bone samples isolated from Charcot's osteoarthropathy patients showed excessive osteoclastic activity in a microenvironment enriched with mediators of bone resorption (IL-1, IL-6 and TNF- $\alpha$ ) [20]. Thus a RANKL-independent pathway, which is also known to play a role in other osteolytic disorders such as rheumatoid arthritis [21] and aseptic loosening [22], could contribute also to the pathogenesis of the Charcot's osteoarthropathy.

This study has indicated, for the first time that the RANKL-dependent pathway is important in the pathogenesis of Charcot's osteoarthropathy, thereby raising the possibility of the use of RANKL inhibition in the management of Charcot's foot. However, our observations also suggest that a RANKL-independent pathway may play a role, but further investigation is required to fully clarify the mechanism involved. If confirmed, specific pharmacological agents that counteract the RANKL-independent pathway, such as anti-TNF strategies, may be useful in the treatment of Charcot's osteoarthropathy. Whatever the relative importance of either pathway, this in vitro technique of generating human osteoclasts from PBMCs may allow specific characterisation of osteoclastic activity in each patient and could, in the future, lead to individually tailored anti-osteoclastic treatment for the patient with acute Charcot's osteoarthropathy.

Acknowledgements G. Mabilleau was supported by Furlong Charitable Trust. N. L. Petrova was supported by Diabetes UK Grant: BDA:05/0003025 and an EFSD/AstraZeneca Clinical Travel Fellowship.

Duality of interest The authors declare that there is no duality of interest associated with this manuscript.

\section{References}

1. Gough A, Abraha H, Li F et al (1997) Measurement of markers of osteoclast and osteoblast activity in patients with acute and chronic diabetic Charcot neuroarthropathy. Diabet Med 14:527-531

2. Teitelbaum SL (2000) Bone resoption by osteoclasts. Science 289:1504-1508

3. Yasuda H, Shima N, Nakagawa N et al (1998) Osteoclast differentiation factor is a ligand for osteoprotegerin/osteoclasto- genesis-inhibitory factor and is identical to TRANCE/RANKL. Proc Natl Acad Sci U S A 95:3597-3602

4. Hofbauer LC, Schoppet M (2004) Clinical implications of the osteoprotegerin/ RANKL /RANK system for bone and vascular diseases. JAMA 292:490-495

5. Jeffcoate W (2004) Vascular calcification and osteolysis in diabetic neuropathy-is RANK-L the missing link? Diabetelogia 47:1488-1492

6. Sabokbar A, Athanasou NS (2003) Generating human osteoclasts from peripheral blood. Methods Mol Med 80:101-111

7. Fujikawa Y, Quinn JM, Sabokbar A, McGee JO, Athanasou NA (1996) The human osteoclast precursors circulate in the monocyte fraction. Endocrinology 137:4058-4060

8. Sanders LJ, Frykberg RG (2001) Charcot neuroarthropathy of the foot. In: Bowker JH, Phiefer MA (eds) Levin \& O'Neal's the diabetic foot. 6th edn. Mosby, St Louis, pp 439-466

9. Flanagan AM, Lader CS (1998) Update on the biologic effects of monocyte-macrophage colony-stimulating factor. Curr Opin Hematol 5:181-185

10. Motoyoshi K (1998) Biological activities and clinical application of M-CSF. Int J Hematol 67:109-122

11. Kobayashi K, Takahashi N, Jimi E et al (2000) Tumor necrosis factor alpha stimulates osteoclast differentiation by mechanism independent of ODF/RANKL-RANK-interaction. J Exp Med 191: 257-286

12. Kudo O, Fujikawa Y, Itonaga I, Sabokbar A, Torisu T, Athanasou NA (2002) Proinflammatory cytokine (TNFalpha/IL-1alpha) induction of human osteoclast formation. J Pathol 198:220-227

13. Kudo O, Sabokbar A, Pocock A, Itonaga I, Fujikawa Y, Athanasou NA (2003) Interleukin-6 and interleukin-11 support human osteoclast formation by RANKL-independent mechanism. Bone $32: 1-7$

14. Bendre MS, Montague DC, Peery T, Akel NS, Gaddy D, Suva LJ (2003) Interleukin-8 stimulation of osteoclastogenesis and bone resorption is a mechanism for the increased osteolysis of metastatic bone disease. Bone 33:28-37

15. Edwards JR, Sun SG, Locklin R et al (2006) LIGHT (TNFSF14), a novel mediator of bone resorption, is elevated in rheumatoid arthritis. Arthritis Rheum 54:1451-1462

16. Hirayama T, Danks L, Sabokbar A, Athanasou NA (2002) Osteoclast formation and activity in the pathogenesis of osteoporosis in rheumatoid arthritis. Rheumatology 41:1232-1239

17. Zou W, Bar-Shavit Z (2002) Dual modulation of osteoclast differentiation by lipopolysaccharide. J Bone Miner Res 17:1211-1218

18. Grimaud E, Soubigou L, Couillaud S et al (2003) Receptor activator of nuclear factor kappaB ligand (RANKL)/osteoprotegerin $(\mathrm{OPG})$ ratio is increased in severe osteolysis. Am J Pathol 163:2021-2031

19. Jeffcoate WJ, Game F, Cavanagh P (2005) The role of proinflammatory cytokines in the cause of neuropathic osteoarthropathy (acute Charcot foot) in diabetes. Lancet 366:2058-2061

20. Baumhauer JF, O'Keefe RJ, Schon LC, Pinzur MS (2006) Cytokine-induced osteoclastic bone resorption in Charcot arthropathy: an immunohistochemical study. Foot Ankle Int 27:797-800

21. Adamopoulos IE, Sabokbar A, Wordsworth BP, Carr A, Ferguson DJ, Athanasou NA (2006) Synovial fluid macrophages are capable of osteoclast formation and resorption. J Pathol 208:35-43

22. Sabokbar A, Kudo O, Athanasou NA (2003) Two distinct cellular mechanisms of osteoclast formation and bone resorption in periprosthetic osteolysis. J Orthop Res 21:73-80 